\title{
Laparoscopic Ligation of a First-Trimester Ovarian Artery Pseudoaneurysm
}

\author{
D. Ashley Hill, MD, Kimberly Liekweg, BA \\ Department of Obstetrics and Gynecology, University of Central Florida College of Medicine, Orlando, Florida \\ (both authors).
}

\begin{abstract}
Introduction: An ovarian artery pseudoaneurysm is a rare disorder of pregnancy.

Case Description: We present a case of an unruptured ovarian artery pseudoaneurysm during the first trimester, managed laparoscopically. The patient had undergone a previous right salpingo-oophorectomy and presented several weeks later with severe right adnexal pain. Doppler sonography and magnetic resonance arteriography revealed a right ovarian artery pseudoaneurysm. The patient declined selective embolization. Laparoscopic ligation of the pseudoaneurysm resulted in resolution of her symptoms. A complete description of the case, radiologic imaging, and a review of the literature are provided.
\end{abstract}

Discussion: Laparoscopy is an option for treating unruptured pelvic pseudoaneurysms during early pregnancy.

Key Words: Aneurysm, Laparoscopy, Ovarian artery, Pregnancy, Pseudoaneurysm.

Citation Hill DA, Liekweg K. Laparoscopic ligation of a first-trimester ovarian artery pseudoaneurysm. CRSLS e2015.00030. DOI: 10.4293/CRSLS.2015.00030.

Copyright (c) 2015 by SLS, Society of Laparoendoscopic Surgeons. This is an open-access article distributed under the terms of the Creative Commons Attribution-Noncommercial-ShareAlike 3.0 Unported license, which permits unrestricted noncommercial use, distribution, and reproduction in any medium, provided the original author and source are credited.

Address correspondence to: D. Ashley Hill, MD, Department of Obstetrics and Gynecology, University of Central Florida College of Medicine. 235 East Princeton Street, \#200, Orlando, FL 32804. Telephone: 407-303-1444; Fax: 407-303-1424; E-mail: d.ashley.hill.md@flhosp.org

\section{CASE REPORT}

A 25-year-old multiparous woman was transferred to our institution because of worsening right lower quadrant pain at 12 weeks' gestation. She had undergone a right salpingoophorectomy at another center 6 weeks before admission, to treat ovarian torsion via a vertical midline incision. Two days before admission, she fell against a table, striking her right groin, and thereafter reported sharp continuous right lower quadrant pain radiating into the right groin. She noted that she did not have pain before the injury. She denied flank pain, dysuria, distention, vomiting, or other symptoms. The patient's medical history included 2 prior cesarean deliveries, with both pregnancies complicated by preeclampsia. She reported that the pain was intolerable and worsening progressively, despite narcotic analgesics.

Physical examination revealed normal vital signs. The abdomen was soft, without distention; tender only in the right lower quadrant; and without masses, bruising, hernias, or costovertebral angle tenderness. There were healed vertical and Pfannenstiel incisions. Bimanual examination revealed a 12-week-sized, nontender uterus with a closed cervix and no obvious pelvic masses, although pain limited examination of the right lower quadrant.

Laboratory findings included a normal white blood cell count of 10.2 and anemia (hemoglobin, 9.1). Results of coagulation studies and serum chemistry testing, including creatinine and liver function, were normal.

Transvaginal ultrasonography revealed a single live intrauterine pregnancy with a gestational age of 12 weeks, 3 days and a $3.6 \times 3.4-\mathrm{cm}$ right adnexal mass. Color Doppler revealed to-and-fro blood flow within the aneurysm sac, where arterial blood flows into the aneurysm cavity during systole, then reverses into the original artery during diastole, consistent with a pseudoaneurysm (Figure 1). A pelvic magnetic resonance angiogram revealed a patent aorta and external and internal iliac vessels and a probable right ovarian artery pseudoaneurysm (Figure 2).

A vascular surgeon suggested embolization, but the patient declined, stating that she preferred surgery. After the 


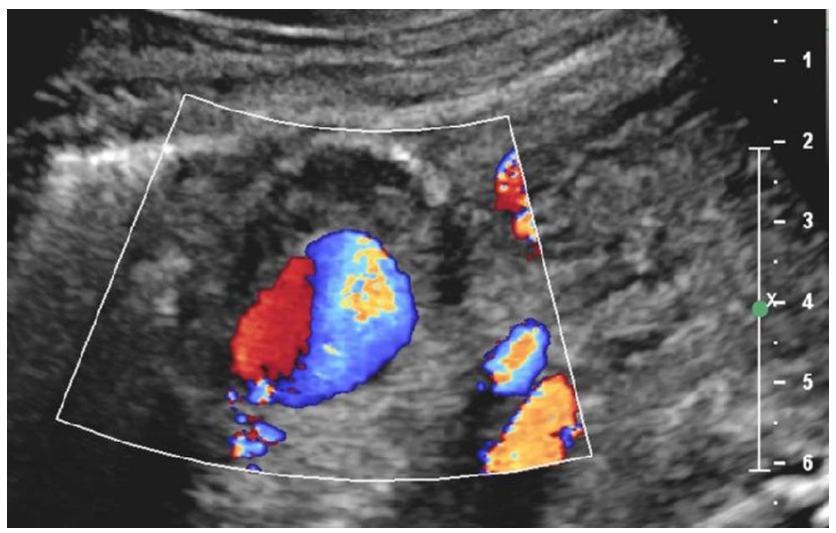

Figure 1. Doppler transvaginal sonogram shows turbulent arterial and venous blood flow within a right ovarian artery pseudoaneurysm sac. Red: arterial flow; blue: venous flow.

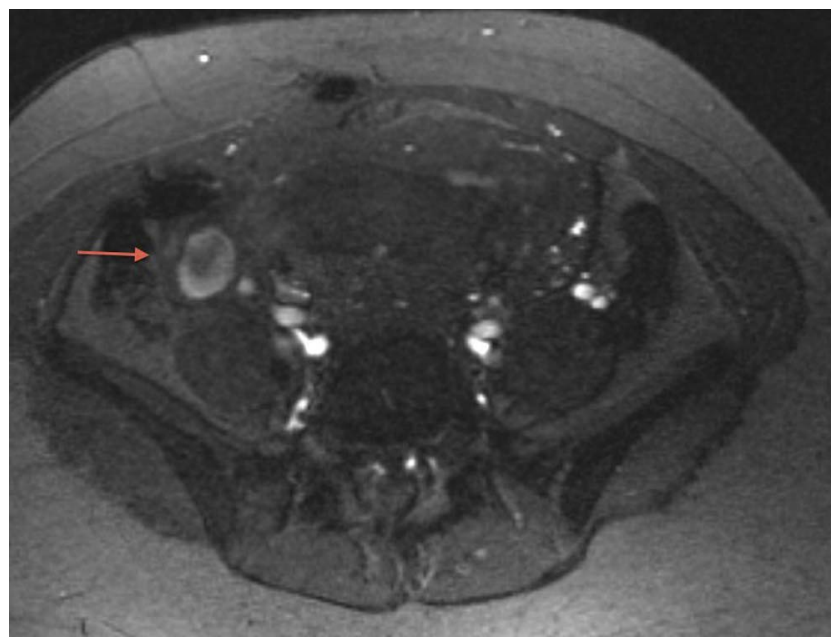

Figure 2. Magnetic resonance angiogram shows a right ovarian artery pseudoaneurysm (arrow).

planned procedure was reviewed with the patient, she underwent laparoscopy.

The abdomen was accessed via a 5-mm optical trocar placed in the left upper quadrant. After insufflation of carbon dioxide, 2 additional 5-mm trocars were placed in the left and right midquadrants. The uterine fundus was adherent to the anterior abdominal wall. The left ovary and fallopian tube were visually normal, whereas the right ovary and fallopian tube were surgically absent. Loops of small intestines were draped over the pseudoaneurysm, which was located along the right pelvic sidewall. There was no hemoperitoneum, retroperitoneal hematoma, or ascites. After enterolysis was performed with a harmonic scalpel and after the peritoneum was opened above and parallel to the right ureter, the ureter was found to be normal in caliber and traveling under the 4-cm mass (Figure 3). With the ureter under direct visualization, the proximal portions of the ovarian artery and vein were dissected from the underlying tissue, and 2 sutures of 0 -polyglactin were passed under the vessels from the lateral to the medial position and tied with an extracorporeal knot pusher. The pseudoaneurysm was excised distal to both ligatures and removed in a specimen bag. Pathologic examination of the mass revealed a pseudoaneurysm with intraluminal thrombosis.

The patient tolerated the procedure uneventfully and was discharged the next day. She reported complete resolution of the right lower quadrant pain by the next morning. She returned for a second cesarean delivery at 34 weeks' gestational age after presenting with preterm premature rupture of membranes. Delivery of the neonate required lysis of dense adhesions between the uterus and abdominal wall. No adnexal adhesions, masses, or vascular abnormalities were identified. There were no postoperative complications and the patient left the hospital 3 days after delivery.

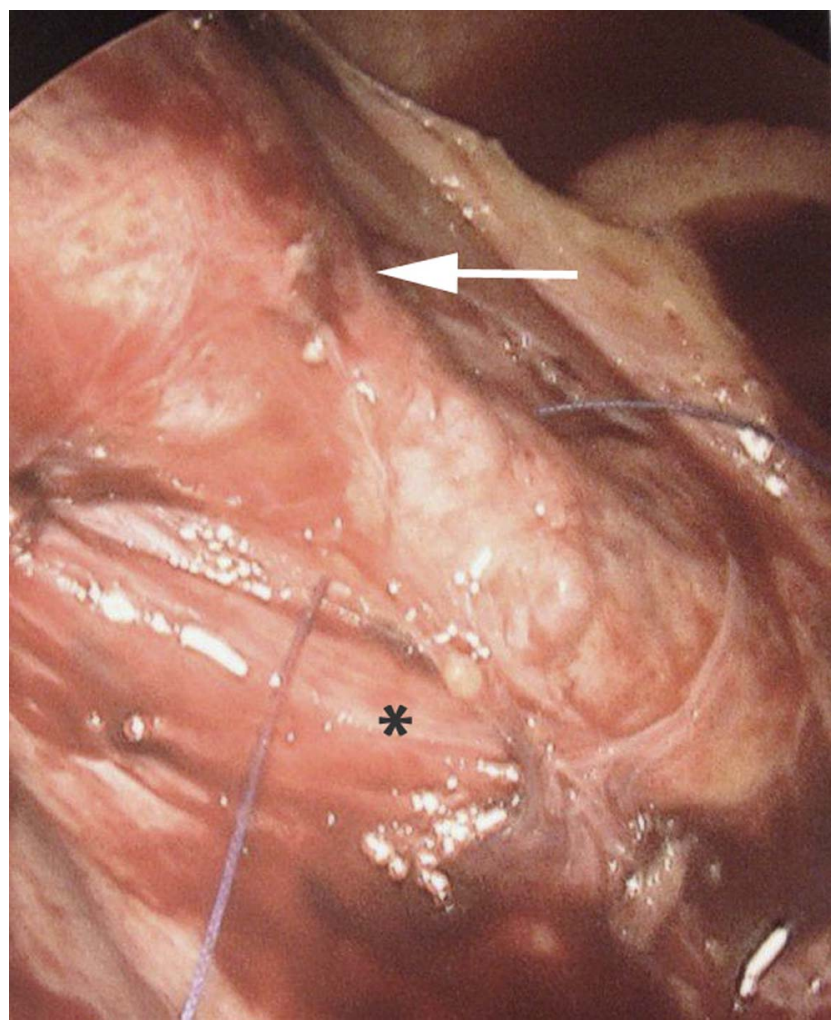

Figure 3. Intraoperative photo of right ovarian artery pseudoaneurysm (arrow) with ballooning of the distal portion of the ovarian artery. The ligature lies between the pseudoaneurysm and ureter (asterisk). 


\section{DISCUSSION}

Pseudoaneurysm is a rare complication of gynecologic surgery and pregnancy. A pseudoaneurysm is a hematoma with flow that occurs when a damaged or weakened arterial wall allows for extravasation of blood outside the vessel lumen, causing turbulent flow. Direct trauma to the arterial wall during surgery or structural changes caused by the hemodynamic and hormonal alterations in pregnancy may weaken vessels, predisposing them to pseudoaneurysm formation. ${ }^{1-2}$ Pseudoaneurysm has been described after many types of gynecologic procedures, including laparoscopic myomectomy, ${ }^{3}$ laparoscopic excision of endometriosis, ${ }^{4}$ hysterectomy, ${ }^{5}$ dilation and curettage, ${ }^{6}$ and cervical conization. ${ }^{7}$ Although cesarean delivery appears to be the most common obstetric procedure leading to pseudoaneurysm, ${ }^{1}$ other events, such as forceps and spontaneous vaginal delivery, may lead to the phenomenon. ${ }^{6}$

Gynecologists should be aware of the possibility of pseudoaneurysm, because it can cause pain or, if rupture occurs, severe or fatal hemodynamic compromise. ${ }^{8}$ Pelvic aneurysm and pseudoaneurysm are usually diagnosed in the third trimester or puerperium. Patients can present with abdominal, pelvic, or flank pain; vaginal hemorrhage due to rupture of the vessel within the uterus; or hemodynamic instability due to retroperitoneal hematoma formation. Clinicians unfamiliar with this condition may attribute symptoms to more common conditions and therefore fail to diagnose the pseudoaneurysm before life-threatening rupture.

Pseudoaneurysm can develop in several different pelvic vessels, although uterine artery pseudoaneurysm appears to be the most common. One group evaluated 588 consecutive cases of postpartum hemorrhage treated with arterial embolization and identified 18 patients with pelvic pseudoaneurysm. 9 Of these 18 cases, 15 involved the uterine artery, 4 the left obturator artery, and 1 right internal pudendal artery (some patients had bilateral arterial involvement).

Ovarian artery true aneurysm is rare. There are fewer than 20 reported cases of ruptured true ovarian artery aneurysm, and although there are a few reports of ruptured ovarian aneurysm remote from pregnancy, most often these develop during the third trimester or puerperium. ${ }^{10-11}$ Ovarian artery pseudoaneurysm is even less common, with only a few reported cases complicating postpartum hysterectomy, ${ }^{12}$ abdominal hysterectomy, ${ }^{13}$ and unilateral salpingo-oophorectomy. ${ }^{14}$ Although preg- nancy appears to predispose patients to pseudoaneurysm, the most likely cause of this patient's pseudoaneurysm was vessel trauma from the salpingoophorectomy, perhaps exacerbated by trauma. We speculate that our patient developed the pseudoaneurysm after removal of her ovary, and the trauma to her abdomen when she fell against the table caused the pseudoaneurysm to enlarge. Unruptured ovarian artery pseudoaneurysm appears to be rare. There is no evidence that a specific size increases the risk of rupture. However, ruptured vascular lesions can cause massive hemorrhage. The patient's escalating pain despite narcotic analgesics increased our concern that the pseudoaneurysm would rupture, leading to our decision to pursue treatment during pregnancy.

Imaging modalities useful for diagnosing ovarian artery pseudoaneurysm include Doppler ultrasonography, angiography, pelvic computed tomography, and magnetic resonance imaging. ${ }^{3}$ Blood flowing through the damaged arterial wall layers typically assumes a to-and-fro pattern (also known as yin-yang flow), because blood flows into the aneurysm cavity during systole then reverses into the original artery during diastole (Figure 1) ${ }^{8}$ Duplex Doppler sonography is relatively inexpensive and noninvasive and has a 95\% sensitivity and specificity for diagnosing pseudoaneurysm. ${ }^{15}$ Early diagnosis is critical because of the potential for rupture.

In the past, laparotomy with vessel ligation was the only treatment option for the ruptured true aneurysm or pseudoaneurysm. Selective arterial embolization is an effective therapy ${ }^{8}$ although hemodynamic instability may necessitate surgery to evacuate an expanding hematoma and ligate a ruptured aneurysm. ${ }^{11}$ Embolization is a wellknown treatment for postpartum hemorrhage. However, this procedure requires that the physician be capable of performing embolization, which may not be available in some facilities. Our patient declined embolization and requested surgery. We decided to pursue laparoscopy because of the patient's hemodynamic stability and relatively small first trimester uterus, as well as the availability of blood products if a hemorrhage developed. Apparently, minimally invasive surgery is an uncommon treatment for ovarian artery vascular abnormalities. We were able to identify 1 other reported case in which a minimally invasive approach was undertaken, where the surgeons used retroperitoneal laparoscopy (lumboscopy) to evacuate a persistent retroperitoneal hematoma that persisted despite selective embolization. ${ }^{16}$

Clinicians should consider pseudoaneurysm as a potential diagnosis when evaluating a patient with hypovolemia; in- 
tractable postpartum hemorrhage; or pelvic, abdominal, or flank pain. Selective arterial embolization is a highly successful procedure for hemodynamically stable patients but may not always be possible or desired. We advise that clinicians consider laparoscopy when selective embolization is not available or is unsuccessful and the patient is hemodynamically stable. Successful laparoscopic treatment requires that surgeons have knowledge of vascular and retroperitoneal anatomy, an ability to visualize the ureter and pelvic sidewall vascular structures, the ability to convert to a laparotomy if necessary, and the availability of blood products.

\section{References:}

1. Isono W, Tsutsumi R, Wada-Hiraike $\mathrm{O}$, et al. Uterine artery pseudoaneurysm after cesarean section: case report and literature review. J Minim Invasive Gynecol. 2010;17:687-691.

2. Barrett JM, Van Hooydonk JE, Boehm FH. Pregnancy-related rupture of arterial aneurysms. Obstet Gynecol Surv. 1982;37:557-566.

3. Asai S, Asada H, Furuya M, Ishimoto H, Tanaka M, Yoshimura Y. Pseudoaneurysm of the uterine artery after laparoscopic myomectomy. Fertil Steril. 2009;91:929.e1-e3.

4. Ferrero S, Bogliolo S, Rossi UG, et al. Unusual complication of excision of pelvic endometriosis: pseudoaneurysm of the left uterine artery. Fertil Steril. 2010;93:264-266.

5. Lee WK, Roche CJ, Duddalwar VA, Buckley AR, Morris DC. Pseudoaneurysm of the uterine artery after abdominal hysterectomy: radiologic diagnosis and magagement. Am J Obstet Gynecol. 2001;185:1269-1272.

6. Soyer P, Fargeaudou Y, Morel O, Boudiaf M, Le Dref O, Rymer R. Severe postpartum haemorrhage from ruptured pseudoaneurysm: successful treatment with transcatheter arterial embolication. Eur Radiol. 2008;18:1181-1187.

7. Zanati J, Sergent F, Clavier E, Marpeau L. Late postconization hemorrhage and false aneurysm of the uterine pedicle [in French]. J Gynecol Obstet Biol Reprod (Paris). 2006;35:725-728.

8. Vijayakumar A, Srinivas A, Chandrashekar BM, Vijayakumar A. Uterine vascular lesions. Rev Obstet Gynecol. 2013;6:69-79.

9. Dohan A, Soyer P, Subhani A, et al. Postpartum hemorrhage resulting from pelvic pseudoaneurysm: a retrospective analysis of 588 consecutive cases treated by arterial embolization. Cardiovasc Intervent Radiol. 2013;36:1247-1255.

10. Kodaira Y, Iwamura T, Hoshino H, Takahashi K, Kawahigashi Y, Matsumoto K. Spontaneous rupture of aneurysms of the ovarian artery at times remote from pregnancy. J Nippon Med Sch. 2014;81:101-105.

11. Chao L-W, Chen C-H. Spontaneous rupture of an ovarian artery aneurysm: case report and review of the literature. Gynecol Obstet Invest. 2009;68:104-107.

12. Rathod K, Deshmukh H, Asrani A, Salvi V, Prabhu S. Successful embolization of an ovarian artery pseudoaneurysm complicating obstetric hysterectomy. Cardiovasc Intervent Radiol. 28:113-116, 2005.

13. Patel T, Liddell R, Hofmann L. Transcatheter embolization of an ovarian artery pseudoaneurysm: an elusive source of posthysterectomy bleeding. EJVES Extra. 2005;10:18-20.

14. Ball R, Picus D, Goyal R, Wilson D, Rader J. Ovarian artery pseudoaneurysm: diagnosis by Doppler sonography and treatment with transcatheter embolization. J Uttrasound Med. 1995;14:250-252.

15. Helvie MA, Rubin JM, Silver TM, Kresowik TF. The distinction between femoral artery pseudoaneurysms and other causes of groin masses: value of duplex Doppler sonography. AJR Am J Roentgenol. 1988;150:1177-80.

16. Guillem P, Bondue X, Chambon JP, Lemaitre L, Bounoua F. Spontaneous retroperitoneal hematoma from rupture of an aneurysm of the ovarian artery following delivery. Ann Vasc Surg. 1999; 13:445-448. 